\title{
Valorización económica de los cuidados no remunerados en salud: un aporte al reconocimiento del trabajo invisible de los hogares y las mujeres*
}

\author{
Determining the Worth of Unpaid Health Care: A Contribution to Recognize the Invisible Work by Women \\ and Homes
}

Valoração econômica dos cuidados não remunerados em saúde: uma contribuição para o reconhecimento do trabalho invisível das famílias e as mulheres

Karina Batthyány Dighiero

Universidad de la República, Uruguay

ORCID: http://orcid.org/0000-0001-6836-9806

Sol Scavino Solari ${ }^{\text {a }}$

Universidad de la República, Uruguay

ORCID: http://orcid.org/0000-0002-6675-7765
DOI: https://doi.org/10.11144/Javeriana.rgps17-34.vecr

Fecha de recepción: 30 Mayo 2017

Fecha de aceptación: 20 Octubre 2017

\section{Resumen:}

El presente artículo tiene como objetivos la valorización del cuidado no remunerado en salud en Uruguay y la caracterización de los/as cuidadores/as (remunerado o no) de personas enfermas. Dichos objetivos son llevados a cabo, principalmente, mediante el análisis cuantitativo de los datos de la primera Encuesta Nacional de Cuidados No Remunerados en Salud (2013) en Uruguay. La encuesta permite conocer y valorizar (método de costo de reemplazo) el tiempo de cuidados no remunerados en salud, así como la caracterización de las/os cuidadoras/es no remunerados en salud. El artículo muestra que el trabajo de cuidados no remunerados en salud realizado por los hogares equivale a un tercio del agregado del sector salud del Producto Interno Bruto y que las mujeres son las principales cuidadoras. En consecuencia, asumen los costos de pérdida de autonomía y limitaciones en el goce de derechos que la realización de este trabajo implica.

Palabras clave: género, cuidados, políticas públicas.

\begin{abstract}
:
This paper is intended to determine the worth of unpaid health care in Uruguay and to characterize the caregivers (either paid or unpaid) who serve ill people. These objectives are accomplished mainly through the quantitative analysis of the data gathered by the first National Survey of Unpaid Health Care (2013) in Uruguay. This survey enables to become aware and set the worth of the unpaid health care times (replacement cost method) and to characterize the unpaid caregivers. This paper shows how the unpaid health care provided by homes accounts for a third of whole health care aggregate in the Gross Domestic Product. Women are the main caregivers, thus assuming the costs of losing their autonomy and seeing reduced the opportunities to enjoy their rights.
\end{abstract}

Keywords: gender, care, public policies.

Resumo:

O presente artigo tem como objetivo a valorização do cuidado não remunerado em saúde em Uruguai e a caracterização dos cuidadores e das cuidadoras (remunerados ou não) de pessoas doentes. Tais objetivos são levados a cabo, principalmente, mediante análise quantitativa dos dados do primeiro Inquérito Nacional de Cuidados Não Remunerados em Saúde (2013) em Uruguai. O inquérito permitiu conhecer e valorizar (método de custo de reposição) o tempo de cuidados não remunerados em saúde, bem como a caracterização das/dos cuidadoras/es não remunerados em saúde. O artigo demonstra que o trabalho de cuidados não remunerados em saúde realizado pelas famílias equivale a um terço do agregado do setor saúde no Produto Interno Bruto e que as mulheres são as principais cuidadoras. Em consequência, assumem os custos de perda de autonomia e limitações no desfrute de direitos que a realização desse trabalho implica.

Palavras-chave: gênero, cuidados, políticas públicas Introducción.

\footnotetext{
a Autora de correspondencia. Correo electrónico: solscavino@gmail.com
} 


\section{Introducción}

El problema de la crisis de cuidado y cómo hacer frente a ellos requiere pensamiento en varios niveles (teórico, metodológico y en el diseño y organización de políticas públicas) y desde varias disciplinas. Dentro de las distintas poblaciones que requieren cuidados, se encuentran las personas enfermas crónicas y agudas. En Uruguay, las instituciones que están mayoritariamente involucradas en los cuidados de las personas enfermas son, hasta ahora, el Sistema Nacional Integrado de Salud y las familias principalmente. Dentro de ellas, como ha sido evidenciado por varios estudios antecedentes, son las mujeres quienes realizan la mayor parte del trabajo de cuidados y asumen costos en cuanto a la participación en la esfera pública y el pleno goce de sus derechos por dedicarse a esta tarea.

En el marco del despliegue del recientemente creado Sistema Nacional Integrado de Cuidados en Uruguay, el presente artículo tiene por objetivo visibilizar la carga de cuidados no remunerados dedicados a personas en situación de dependencia por enfermedades agudas o crónicas. A su vez, pretende aportar a la discusión sobre la interacción entre el sistema sanitario y de cuidados. En este marco, se valoriza económicamente el cuidado no remunerado en salud que llevan a cabo las familias, con el fin de poder discutir en términos sociales y económicos qué implicancias tiene una posible redistribución del trabajo de cuidado.

La consideración del trabajo no remunerado (que incluye la realización de las tareas domésticas, de cuidado, de apoyo a otros hogares y de voluntariado) como un tipo de trabajo similar al remunerado, ha permitido volver visible el aporte de los hogares y de las mujeres a las sociedades en su conjunto y las economías nacionales. Con base en esta perspectiva, se han analizado los datos de la Encuesta Nacional de Cuidados No Remunerados en Salud que permiten conocer el tiempo que las personas y los hogares uruguayos dedican a cuidar de manera no paga de personas enfermas. El análisis consta de dos grandes partes: la primera es de tipo descriptiva (se complementa con los datos de la Encuesta Continua de Hogares de 2013) y desarrolla las características sociodemográficas de los cuidadores remunerados y no remunerados en salud. En segundo lugar, se lleva a cabo un ejercicio de valorización económica del trabajo no remunerado, siguiendo la metodología de costo de reemplazo para poder estimar a qué porcentaje del PIB se asemeja el monto de trabajo que no está siendo considerado por aquel, así como cuánto del agregado del sector salud en el PIB representa el trabajo que se realiza de manera invisible, no paga por parte de las mujeres en el marco de los hogares uruguayos.

El estudio muestra no solo que las mujeres son la mayoría de las cuidadoras (remuneradas o no) y que dedican más tiempo promedio que los varones a dichas tareas, sino que, si se imputara un valor económico al tiempo dedicado por estas personas al cuidado en salud, este representaría un 1.8\% del PIB nacional, y un tercio del agregado del sector salud.

Es decir, que si se considera que la producción del Estado y el mercado de bienes y servicios de salud es suficiente para cubrir el cuidado en salud de la población, se estaría obviando casi un tercio de su producción proveniente del trabajo gratuito de las mujeres en el marco de los hogares. La visibilización del aporte de los hogares y de las mujeres al cuidado en salud pone sobre la mesa el cuestionamiento acerca de cómo debería ser una interrelación virtuosa y corresponsable entre los distintos sectores (Estado, mercado, comunidad y hogares) y actores (varones y mujeres) para que estos nos sean fuente de desigualdades sociales de género.

\section{Cuidados como trabajo y su aporte central al bienestar social}

El cuidado es un concepto en discusión, lo cual permite considerar una serie variada de tareas, actividades, dimensiones y lugares donde ocurre. Dentro de las definiciones más amplias de cuidado, se encuentra que este es una actividad que incluye todo lo que podemos hacer para mantener, continuar y reparar nuestro mundo (cuerpos, ser, ambientes) para vivir en él lo mejor posible [1]. 
En cuanto a las definiciones más operativas, orientadas a la investigación y al diseño de políticas públicas, se caracteriza al cuidado como la acción de ayudar a un niño/a o persona en situación de dependencia en las actividades de su vida cotidiana para lograr un mayor bienestar e implica tres grandes dimensiones, hacerse cargo del cuidado material, del costo económico del cuidado y de las emociones y afectividad puestas en juego en el vínculo [2].

Esta definición de cuidados permite pensar que estos pueden ser realizados en el ámbito familiar o estatal/ mercantil por medio de instituciones y servicios, así como pueden ser remunerados o no remunerados. En estos distintos ámbitos, al ser el cuidado una tarea particularmente compleja puesto que implica una parte vincular y afectiva, al ser mercantilizado, la dimensión emocional y vincular también cobra significados especiales que lo distinguen de otro tipo de trabajos, exigiendo, por ejemplo, el desarrollo de una ingeniería emocional por parte de los/as cuidadores/as [3].

Que el cuidado implique costos de tiempo, materiales y económicos permite pensar que se trata de un trabajo la mayor de las veces no remunerado. Así, en el marco de las relaciones sociales del cuidado se producen bienes y servicios que garantizan el bienestar de la población y el sostenimiento de su vida.

Dentro de las distintas tareas de las que las personas (mayoritariamente las mujeres) se hacen cargo en el marco de los hogares no se encuentra solo el cuidado, sino que también se encuentran las tareas domésticas y de trabajo voluntario. Las mujeres son entonces grandes productoras de bienes (alimentos, elaboración de vestimenta, por ejemplo) y servicios (de cuidados, limpieza, entre otros) que son dirigidos a los otros integrantes del hogar y por el que no reciben ningún tipo de reconocimiento (ni social ni económico).

Dicha producción de bienes y servicios había sido obviada en los primeros estudios de los Estados de bienestar social, donde se consideraban como actores productores de bienestar solamente al Estado y al mercado, sectores que durante gran parte de los siglos XIII a XIX en Occidente estuvieron reservados casi exclusivamente para los varones.

A partir de un largo proceso en el cual el pensamiento feminista, a través de las disciplinas como la sociología, la antropología y la economía, logró mostrar que las conceptualizaciones sobre el trabajo, la organización de la producción y los aportes al bienestar eran restringidas a un solo tipo de trabajo (remunerado), los análisis de Estados de bienestar comenzaron a considerar a los hogares como productores de bienes y servicios para la generación y el sostenimiento del bienestar social [4].

Dentro de los aspectos que fueron destacados por el pensamiento feminista sobre las falencias de los análisis de bienestar social anteriores, se destaca que estos tenían un sesgo mercantilista (ya que no se consideraba una gran producción solo por no ser intercambiada en el mercado de empleo) y patriarcal (ya que se invisibilizaba el trabajo de cuidados y doméstico que es realizado por las mujeres, quienes son consideradas "inactivas" cuando no forman parte del mercado de empleo formal y, sin embargo, son activas si se considera que con la realización de tareas domésticas y cuidados están produciendo bienes y servicios fundamentales para la supervivencia) [5].

Una vez que quedó en evidencia la restricción del viejo concepto de trabajo (como aquella actividad por la que se recibe alguna remuneración a cambio - monetaria o en especie-, desconociendo y desvalorizando las tareas realizadas gratuitamente - por amor- en un mundo doméstico, de puertas adentro [6,7], se planteó una nueva definición que establece que el trabajo es cualquier actividad física o mental que transforma al mundo material de manera que sea más útil, que extiende el conocimiento humano y provee y distribuye servicios para los demás [8].

La ampliación y revisión conceptual del trabajo fue acompañada de un cambio en la noción misma de producción, que pasó a ser considerada una actividad en la que un agente económico suministra bienes y servicios a otras unidades, mediante la utilización de insumos, lo cual implica que al igual que en la economía de mercado, los servicios no remunerados para los propios miembros del hogar (domésticos y de cuidado de personas), servicios no remunerados para otros hogares y para la comunidad y servicios voluntarios no remunerados forman parte de la frontera general de producción [9]. 
A partir de estas redefiniciones conceptuales, se ha planteado que existe una organización social del cuidado cuya caracterización depende de la carga de cuidado que tienen sectores como el Estado, el mercado, la comunidad y los hogares, así como de la carga de cuidado que tienen los varones y las mujeres [10].

Autoras como Daly y Lewis [11] han planteado que a partir del cuidado se pueden clasificar distintos regímenes de bienestar social. Por ejemplo, se ha distinguido entre estados familistas, en los cuales la responsabilidad del bienestar recae fundamentalmente sobre las mujeres de las familias, y los desfamiliarizadores en los que hay una derivación de funciones de cuidado, tareas domésticas hacia el Estado y el mercado [12].

Los estudios sobre regímenes de bienestar en América Latina en comparación con otras regiones han mostrado que en la región son preponderantes los Estados de bienestar del tipo familista, que lejos de estimular una nueva organización social del cuidado entre Estado, mercado, familias y comunidad, cuestionan poco el rol de las mujeres en el trabajo no remunerado de cuidados, cuando no se basan en este para la aplicación de las políticas públicas y la generación de productos necesarios para el bienestar de la población [13].

En Uruguay, varios estudios nacionales han mostrado que las familias, particularmente las mujeres dentro de estas, son las principales proveedoras de cuidados y que dicho trabajo es no remunerado, por lo cual las mujeres no acceden a ningún tipo de derecho (por ejemplo, al sistema sanitario, a realizar aportes en una caja jubilatoria, o percibir algún tipo de remuneración u otra fuente de reconocimiento por su realización) [14, 15]. Por esto, la organización social del cuidado se plantea como un desafío para la sociedad uruguaya en la medida que se busque garantizar el derecho al cuidado y avanzar en la equidad de género.

\section{Cuidados como trabajo no remunerado femenino}

Entre los elementos en común que se encuentran en varias definiciones de cuidado se destaca que este es una tarea realizada mayoritariamente por mujeres, en el marco de los hogares y de manera no paga, aunque su mercantilización ha generado un espacio laboral (en América Latina, de características precarias, cuando no informales) en el que se insertan mayoritariamente mujeres [16].

La feminización de los cuidados es producto de la construcción social de género en torno al sexo femenino. El concepto género permite reconocer las asociaciones y construcciones socioculturales sobre los roles y los mandatos impuestos a las personas en función de su sexo biológico a lo largo de la historia [17]. En el marco de las familias nucleares, una división de roles y tareas asignadas a varones y mujeres hizo que estas fuesen identificadas durante gran parte de la historia occidental con las responsabilidades del hogar y como "cuidadoras por naturaleza" de las personas dependientes [18, 19].

La mencionada feminización del cuidado se sostiene por medio de las representaciones sociales del cuidado, y los estereotipos están presentes en las prácticas cotidianas, en las instituciones (escuela, trabajo, familia, sistema sanitario entre otras), en leyes y también en las ideas que los Estados han tenido sobre los roles de varones y mujeres que se reflejan en el diseño de las políticas públicas [20].

En el Uruguay actual, dentro de los tipos de trabajo no remunerados (trabajo doméstico, de cuidados, voluntariado), el de cuidados ha cobrado una especial relevancia ya que está manifestándose como un problema social en la medida en que hay mayor demanda de cuidados que personas que puedan cuidar.

Esto se vincula a una serie de factores demográficos, económicos y socioculturales que han provocado transformaciones sociales como que las mujeres se incorporaran masivamente al mercado laboral, que aumentara el número de familias nucleares, monoparentales y unipersonales, que aumentaran los divorcios, que aumentara la población envejecida en general y en situación de dependencia en particular. Este panorama ha provocado una crisis de cuidado [21], ya que las tensiones de articulación trabajo-familia que las mujeres 
afrontan cada vez son más costosas para ellas y cada vez hay menor oferta de cuidados no remunerados que las mujeres ofrecen para hacer frente a la demanda de la población en situación de dependencia.

El cuidado feminizado y no remunerado representa una limitante para el ejercicio de la ciudadanía social de las mujeres. Por esto, el cuidado en cuanto derecho pone en tela de juicio la actual organización social del cuidado que es familiarista, feminizada y no remunerada e interpela al Estado y la sociedad en su forma de distribuir este trabajo y dar garantías de su cumplimiento. Además, pone en primera plana el cuestionamiento sobre la posición de las mujeres en la sociedad en términos de autonomía y equidad en los distintos ámbitos sociales.

La identificación del concepto de trabajo no remunerado como trabajo habilitó el surgimiento de metodologías para su medición [22]. Así, se comenzó a dar cuenta de cuánto aporta la esfera privada o de la "reproducción humana" a la producción de bienes y servicios comercializados en el mercado [23].

Las encuestas de uso del tiempo fueron uno de los instrumentos más importantes para poder medir el trabajo de cuidados, si bien se encuentran algunas limitaciones en ellas como el hecho de que parten del supuesto de que el tiempo es lineal y objetivo [24], lo cual impide conocer el tiempo que se dedica, por ejemplo, a pensar en las tareas de cuidado que hay que realizar, así como pueden registrarse tiempos dobles por la superposición de tareas (se puede realizar cuidados como acompañamiento y juego a la vez de estar vistiendo a un niño).

En Uruguay, la última encuesta de uso del tiempo (módulo de la Encuesta Continua de Hogares del Instituto Nacional de Estadística, año 2013) muestra que el 90\% de las mujeres participan del trabajo no remunerado (tareas domésticas, de cuidado, de voluntariado y ayuda a otros hogares) y que le dedican un promedio de 37.5 horas semanales, mientras que el $76 \%$ de los varones lo hace, con un promedio de 19.5 horas semanales dedicadas a ello. Dentro de los hogares con presencia de personas dependientes (niños/as de cero a doce años, personas discapacitadas o mayores en situación de dependencia), el 79.7\% de las mujeres se involucra en este tipo de trabajo, con un promedio de veintidós horas semanales, mientras que los varones lo hacen en un $58.8 \%$ y dedican diecisiete horas semanales. Es decir, que cuidan casi un $21 \%$ más de mujeres que de varones y dedican en promedio cinco horas más que ellos a dichas tareas [25].

Dentro de los diferentes tipos de cuidado de los cuales las mujeres se hacen cargo, se encuentran los cuidados a la primera infancia, a personas mayores y discapacitadas en situación de dependencia y los cuidados a personas que transitan enfermedades crónicas o agudas. La dependencia se puede establecer como la falta de autonomía (poder realizar por si solo las actividades de la vida cotidiana), por lo que se necesita la ayuda de otro para lograr un buen estado de salud [26].

Sin embargo, las encuestas de uso del tiempo y el diseño del actual Sistema Nacional Integrado de Cuidados han omitido la consideración de la población que brinda y recibe cuidados no remunerados en salud. El cuidado dirigido a personas enfermas puede ser demandado durante todo el ciclo de vida, principalmente si se piensa en las enfermedades agudas que pueden aparecer en cualquier momento. También sucede que hay enfermos crónicos, que no son considerados en las EUT y por el sistema de cuidados porque, por ejemplo, tienen la edad comprendida entre 13 y 64 años.

Se ha destacado, pensando en los cuidados en salud, que las reformas de los servicios sanitarios ponen un mayor énfasis de la atención a la salud en el entorno de los pacientes y esto genera que el hogar se convierta en un escenario donde confluyen la asistencia del sistema sanitario con los cuidados no remunerados, lo cual está acompañado de un desplazamiento de más cuidados y más complejos, que las mujeres de manera no remunerada realizan en las familias [27].

En un momento donde se ha realizado una fuerte reforma del sistema sanitario uruguayo y en donde se plantea el despliegue de un sistema de cuidados, parece fundamental conocer quiénes se responsabilizan por el cuidado en salud y en qué medida el mismo remunerado o no remunerado, que costos genera para los/ as cuidadores/as y en términos monetarios, cuál es la magnitud del trabajo no pago, si este fuese ofrecido al mercado de empleo, en relación con el PIB nacional y con el agregado del sector salud. 


\section{Cuidados no remunerados en salud: operacionalización y valorización}

Para poder avanzar en la medición de los cuidados no remunerados en salud, se ha planteado una definición de estos que busca considerar el cuidado desde una perspectiva social y no solo como el resultado exclusivo de procesos médicos realizados por profesionales del área (médicos/as, enfermeros/as entre otros) en el marco de las instituciones de salud.

Existe una historia de tensión entre los planteos sobre qué es salud, qué es enfermedad y qué son cuidados en salud desde el modelo médico rehabilitador y el modelo social.

Los planteos del primero se basan en una definición de salud como deficiencia por causas fisiológicas o médicas que pueden ser o no reparables en función de las herramientas de la medicina. Los del segundo, hacen hincapié en que la salud es construida en sociedad (al igual que la enfermedad) y esto se expresa en función de cómo la comunidad expone a riesgos en salud, cuida o no cuida, genera servicios y administra información.

La definición de salud de la Organización Mundial de la Salud (OMS), la cual data de 1946 y que establece que esta es el máximo nivel alcanzado de bienestar físico, psíquico y social, intenta conciliar los modelos anteriormente descritos. Sin embargo, presenta dificultades, ya que lograr un máximo nivel de bienestar físico, psíquico y social es una meta inalcanzable e imposible de ser contrastada empíricamente [28, 29].

Desde entonces se ha avanzado en otras definiciones como que la salud es un estado de bienestar físico, mental y social, con capacidad de funcionamiento y no únicamente ausencia de enfermedades y que integra componentes objetivos y subjetivos, y otras que plantean que la capacidad de funcionamiento implica poder trabajar si se es adulto, estudiar si se es joven, relacionarse con semejantes y gozar de la vida en comunidad [30]. Actualmente existe cierto grado de acuerdo en que el estado de salud o bienestar de una persona es el resultado de la combinación de varios componentes: las funciones y estructuras corporales, actividades y participación y factores contextuales. Los determinantes del estado de salud son entonces las funciones y estructuras corporales (cambios fisiológicos o anatómicos), la participación (capacidad de realizar de la actividad y la realización efectiva de esa actividad) y el contexto con el que interactúa (factores ambientales o personales/psicológicos) [31].

Así, a partir de la base de que quienes necesitan cuidados en salud son aquellas personas que tienen su capacidad de funcionamiento disminuida debido a enfermedades crónicas o agudas, se toma a continuación una propuesta de definicición de cuidados no remunerados en salud como:

"[...] todas las tareas destinadas al cuidado en el ámbito de la salud realizadas para los miembros del hogar que no pueden hacerlas por sí mismos, sin recibir pago alguno por ellas. También incluyen a los que no viven en el hogar y ya sean familiares o no. Por personas que no pueden realizar por sí mismas las actividades de cuidado de salud no remunerado nos referimos a niños/as y niñas/as, ancianos/as dependientes, personas con discapacidad inhabilitados para autocuidarse, enfermos crónicos, de episodios agudos inhabilitantes, terminales, etc. [32]"

Las autoras operacionalizan cuatro grandes tipos de cuidados: los específicos (tareas como hacer tratamientos como curaciones, inyecciones u otros), los de apoyo (elaboración de alimentos o tareas de higiene que responden específicamente ante una situación de enfermedad), acompañamiento (a consultas médicas, traslados, entretenimiento) y de gestiones con el sistema sanitario (solicitud de consultas, retiro de análisis).

Estas se basan en investigaciones realizadas anteriormente en España, Canadá y Chile, principalmente, donde se han estudiado los cuidados en salud provistos por las familias ante la notoria falencia de los servicios públicos en garantizarlos [33].

Como resultados principales encuentran que, en efecto, son las mujeres en el marco de las familias las principales fuentes de cuidados en salud. Por ejemplo, solo el $12 \%$ del tiempo que se dedica al cuidado en salud es brindado por los profesionales sanitarios, mientras que el $88 \%$ restante es tiempo de trabajo familiar no remunerado, y el sistema sanitario es tan solo la punta de un gran iceberg de trabajo aportado para la salud 
de la población, del cual los hogares y particularmente las mujeres que los integran son la base invisible [34, $35,36]$.

En los antecedentes se destaca que las mujeres tienen una participación mayoritaria en los cuidados no pagos en salud en relación con los varones y que son mayoritariamente mujeres de 45 años y más, de niveles económicos medios bajos, con poco contacto con el mercado de empleo. Gran parte de los estudios antecedentes concuerdan en que son las mujeres que aportan más tiempo de trabajo de cuidados no remunerados en salud $(37,38,39,40,41)$. Este fenómeno es observado una y otra vez para todos los componentes del trabajo no remunerado.

Dentro del trabajo de cuidados, los estudios en la región sobre cuidados no remunerados en salud son escasos, ya que hasta el momento Chile, Colombia y Uruguay son los países que han realizado estudios exploratorios en la temática. La escasez de información y datos confiables sobre el papel que las familias cumplen en el cuidado de la salud tiene repercusiones negativas en la formulación y planificación de políticas de salud [42]. La necesidad de aplicar o crear indicadores que den cuenta del aporte que las familias realizan al cuidado de la salud de sus miembros es un desafío latente en la región. En Uruguay se ha realizado un estudio en el que se llevó a cabo la primera Encuesta Nacional de Cuidados No Remunerados en Salud del 2013, representativa de las localidades de 5000 habitantes y más, que muestra que la mayoría de las cuidadoras no remuneradas en salud son mujeres $(74.4 \%)$ y que cuidan en promedio doce horas semanales más que los varones (57 vs. 46) [43].

En este marco, en el que el trabajo no remunerado de cuidados en salud es realizado principalmente por mujeres, invisible a través de las estadísticas oficiales, debido a que carece de valor social y económico, es que se ha planteado la inquietud de conocer qué valor económico podría tener dicho trabajo para cuestionar la participación y reorganización de distintas instituciones en Uruguay para hacer frente a las necesidades de cuidado y garantizar el cumplimiento de estos en cuanto derecho.

La valorización del trabajo no remunerado implica una profunda revisión de la teoría económica, y existen discusiones sobre los elementos a favor y en contra de la realización de estos ejercicios [44]. Dentro de los aspectos positivos de elaborar cifras que permitan resignificar lo productivo incorporando el trabajo no remunerado, se encuentra la visibilización de trabajo oculto y un cuestionamiento a la economía como el estudio de asignación de recursos escasos entre fines limitados, para plantear una "ciencia del aprovisionamiento de la vida humana" [45].

En Uruguay se han realizado ejercicios de valorización económica del trabajo no remunerado que han mostrado que la producción de los hogares representa los 26.6 puntos porcentuales del PIB nacional en 2009 y 22.9 en $2013[46,47]$.

En esta línea, nos proponemos a continuación presentar algunos resultados de la valorización del cuidado no remunerado en salud realizado mayoritariamente por las mujeres desde los hogares en Uruguay, para poder compararlo con otras medidas económicas y sociales. Para colaborar con su valorización y con la resignificación del papel de los hogares y las mujeres en el sistema de bienestar, el presente artículo se propone entonces conocer cuál es el valor económico que representa el cuidado no remunerado en salud, además de cuáles son las características de las personas que lo llevan a cabo, con el fin de discutir el papel de las familias (varones y mujeres), el Estado y el mercado en la provisión de bienes y servicios para el cuidado en salud de personas enfermas crónicas y agudas.

Finalmente, la visibilización y valorización del trabajo no remunerado de cuidados permite reflexionar sobre la organización social del cuidado y la participación de diversas instituciones en este, así como develar las relaciones de poder que subyacen al cuidado y que perpetúan desigualdades de género, socioeconómicas, étnico-raciales entre otras. 


\section{Objetivos y metodología}

El objetivo general de este trabajo es realizar una valorización económica del trabajo de cuidados no remunerados en salud que brindan los hogares y personas uruguayas ante las necesidades de personas enfermas crónicas o agudas. También busca caracterizar a las personas cuidadoras, con base en el antecedente de investigación directo que se refleja en el texto de Batthyány, Perrotta y Genta publicado en 2015 [48].

Para ello, se plantea una metodología de análisis cuantitativo de la primera Encuesta Nacional de Cuidados No Remunerados en Salud (ENCNRS), llevada a cabo por el grupo de Sociología de Género de la Facultad de Ciencias Sociales de la Universidad de la República en 2013.

La ENCNRS contó con una muestra bietápica (estratificada por nivel socioeconómico) en la cual se identificó a los hogares que realizan cuidados no remunerados en salud en zonas urbanas de más de 5000 habitantes. Se encuestó a 1198 hogares y se detectó la presencia de cuidados no remunerados en salud en 300 hogares. En estos se indagó acerca de quiénes cuidan dentro de esos hogares, cuánto tiempo se dedica a cada una de las actividades definidas, si los hogares cuentan o no con otros servicios para llevar adelante ese cuidado en salud, cuáles son los costos asociados al cuidado no remunerado en salud por parte de las personas cuidadoras, cuál es el nivel socioeconómico de esos hogares, el tipo de hogar, la región del país a la que pertenecen.

Para la valorización del trabajo no remunerado, otorgándole un valor económico para poder comparar su volumen con los productos y servicios medidos dentro de la economía del mercado, se ha optado por seguir la metodología input por costo de reemplazo que estudios antecedentes en el país han utilizado para realizar ejercicios similares $[49,50]$. Esta se trata de estimar un valor hora promedio que el mercado otorga al cumplimiento de tareas similares a las que se realizan de manera no remunerada e imputar dicho valor a las horas dedicadas al cuidado no remunerado en salud, para luego multiplicarlo por la sumatoria de estas. Así se obtiene una masa salarial plausible de ser contrastada con otros agregados.

\section{Valorización del trabajo de cuidados no remunerados en salud por medio de la metodología de imputar un valor asociado al costo de reemplazo}

Tal y como se viene exponiendo, el presente estudio, para la valorización del trabajo de cuidados no remunerado en salud, ha seguido las propuestas del método input por costo de reemplazo. Se tomó el valor hora promedio, con base en la Encuesta Continua de Hogares 2013, de aquellas personas cuya ocupación principal era o bien ser profesionales de nivel medio de la salud (nivel 3 en codificador internacional de ocupaciones Ciuo 08), trabajadores de los cuidados personales (nivel 5) o limpiadores, asistentes y ayudantes para la preparación de alimentos (nivel 9, categorías ocupacionales más bajas).

Se estudiaron los tipos de tareas integradas en cada una de las categorías que aparecen en el Listado Nacional de Descripción de ocupaciones, codificador Ciuo 08 y se decidió trabajar principalmente con los integrantes del código 5322, trabajadores de cuidados personales a domicilio (tabla 1). 
TABLA 1

Ocupaciones en los subgrupos considerados para la valorización del trabajo no remunerado de cuidados en salud

\begin{tabular}{|c|c|}
\hline $\begin{array}{c}\text { Trabajador de cuidados personales a } \\
\text { domicilio (5322) }\end{array}$ & $\begin{array}{c}\text { Trabajador de cuidados personales en } \\
\text { instituciones (5321) }\end{array}$ \\
\hline $\begin{array}{c}\text { Trabajadores de cuidados personales a } \\
\text { domicilio }\end{array}$ & Cuidador de casa de salud \\
\hline Camillero de ambulancia (domicilio) & $\begin{array}{c}\text { Trabajador de cuidados personales en casas de } \\
\text { salud }\end{array}$ \\
\hline Auxiliar de enfermeria (domicilio) & $\begin{array}{c}\text { Cuidador de enfermos (hospital o sanatorios) } \\
\text { internados de rehabilitación }\end{array}$ \\
\hline $\begin{array}{c}\text { Acompañante de personas convalecientes a } \\
\text { domicilio }\end{array}$ & Auxiliar de enfermería (clínica u hospital) \\
\hline Cuidador de enfermos a domicilio & Camillero de hospital o sanatorio \\
\hline
\end{tabular}

Fuente: elaboración propia con base en el Listado Nacional de Descripción de ocupaciones, codificador Ciuo 08 en la página del Instituto Nacional de Estadística: http://www.ine.gub.uy

Se consideró que las tareas descritas en cada uno de estos trabajos remunerados fuesen homologables a los medidos como cuidados no remunerados en salud en la ENCRS, por el tipo de tareas que se realizan (similares a las presentadas más arriba en la definición de cuidados no remunerados en salud) y porque el ámbito de desarrollo de estas es el espacio doméstico y no uno de tipo institucional u empresarial, lo cual determina similares condiciones de desarrollo del trabajo entre aquellos cuidadores remunerados y no remunerados (tabla 2).

TABLA 2

Resumen del proceso de valorización de cuidados no remunerados en salud

\begin{tabular}{|c|c|c|c|}
\hline ¿Qué se mide? & ¿Cómo se mide? & $\begin{array}{c}\text { ¿Con base en qué puede } \\
\text { ser medido? }\end{array}$ & $\begin{array}{c}\text { ¿En qué se } \\
\text { traduce? }\end{array}$ \\
\hline $\begin{array}{c}\text { Costo de reemplazo de un } \\
\text { trabajador de servicios de } \\
\text { cuidados en salud } \\
\text { generalizado. }\end{array}$ & $\begin{array}{c}\text { Se identifica un cluster de } \\
\text { trabajadores de la salud } \\
\text { que realizan actividades de } \\
\text { servicios en salud. Se } \\
\text { miden sus ingresos. Se } \\
\text { toma la media ajustada. }\end{array}$ & $\begin{array}{c}\text { Encuesta Continua de } \\
\text { Hogares de 2013. Se } \\
\text { establecen las ocupaciones } \\
\text { de cuidados domiciliarios }\end{array}$ & $\begin{array}{c}\text { Un valor asignado a } \\
\text { una hora de trabajo, } \\
\text { plausible de ser } \\
\text { imputado a las } \\
\text { horas de trabajo no } \\
\text { remunerado que se } \\
\text { miden en los } \\
\text { hogares. }\end{array}$ \\
\hline \hline
\end{tabular}

Fuente: elaboración propia.

Un primer paso para conocer el aporte de los hogares al sistema sanitario es calcular el peso del trabajo, al que se le otorgó un valor económico sobre el PIB nacional en 2013 (año en que se realizó la ENCNRS).

Se calculó el valor promedio de la hora de trabajo de aquellas personas que trabajan en los cuidados domiciliarios en salud dentro del mercado de empleo. Este es de sesenta pesos uruguayos por hora.

Este valor es transportado a las horas dedicadas al cuidado de la salud en los hogares, pero por el cual no se recibe remuneración a cambio. Dicho valor se estima para todo un año de trabajo. La cantidad de hogares que realizan cuidados en salud no remunerados se supone constante y se obtiene un valor salarial total como resultado de la sumatoria y multiplicación de las horas monetarizadas. Si se considera el valor hora promedio imputado a las horas que dedican los cuidadores en salud que realizan su trabajo a domicilio, se puede 
establecer que la magnitud de trabajo de cuidados no remunerados en salud, valorizados económicamente, representa un $1.8 \%$ del PIB del país (tabla 3).

TABLA 3

Porcentaje del producto interno bruto que representan los cuidados no remunerados en salud, según el precio promedio de los trabajadores de cuidados personales a domicilio

\begin{tabular}{cc}
\hline \multicolumn{2}{c}{ Porcentaje del PIB nacional $^{1}$} \\
\hline Remunerados (con base en & No remunerados (con base \\
la ECH 2013) & en la ENCNRS) \\
\hline 0.098 & 1.88 \\
\hline
\end{tabular}

Fuente: elaboración propia con base en los datos de la ENCNRS 2013 y la ECH 2013 (INE).

A su vez, si se estima cuál es el aporte de los cuidadores remunerados en salud a domicilio, se constata que este es casi un 5\% del que brindan los no remunerados, lo cual permite afirmar que, ante los servicios que puedan estar ofreciendo el Estado o el mercado para los cuidados en salud a nivel domiciliario, el papel y aporte de los hogares es absolutamente preponderante.

Esta afirmación se vuelve aún más robusta, cuando se considera el hecho de que el aporte de los hogares que brindan cuidados no remunerados en salud sobre el agregado del sector salud es el $32 \%$ de este (tabla 4 ). Si se observan los trabajadores de cuidados personales a domicilio, podemos ver que en el caso de los remunerados representan el $1.7 \%$ del agregado del sector salud. 
TABLA 4

Porcentaje del agregado del sector salud que representan los cuidados no remunerados en salud, según el precio promedio de los trabajadores de cuidados personales a domicilio, en instituciones y ambos

\begin{tabular}{|c|c|c|}
\hline \multicolumn{2}{|c|}{} & $\begin{array}{c}\text { Sobre el agregado del } \\
\text { sector salud del PIB }\end{array}$ \\
\hline \multirow{2}{*}{ Cuidadores remunerados } & 5322 (domiciliarios) & 1.7 \\
\cline { 2 - 3 } (ECH 2013) & 5321 (institucionales) & 3 \\
\cline { 2 - 3 } & Ambos (5322 y 5321) & 4,7 \\
\hline \multirow{2}{*}{ Cuidadores no } & 5322 (domiciliarios) & 32 \\
\hline \multirow{2}{*}{ remunerados (ENCNRS } & 5321 (institucionales) & 50 \\
\cline { 2 - 3 } 2013$)$ & Ambos (5322 y 5321) & 40 \\
\cline { 2 - 3 } & & \\
\hline
\end{tabular}

Fuente: elaboración propia con base en los datos de la ENCNRS 2013 y la ECH 2013 (INE).

Así, los hogares generan el valor de un tercio de lo que generan el Estado y el mercado en bienes y servicios para la salud de la población. Esto implica que, de no existir el trabajo no remunerado de los hogares y las mujeres dentro de estos, el Estado y el mercado deberían producir al menos un tercio más de bienes y servicios en relación con la producción actual para satisfacer las necesidades de la población.

\section{Características de los cuidados no remunerados en salud y cuidados remunerados en salud}

El cuidado domiciliario en salud está altamente feminizado, ya que sin importar si es remunerado o no remunerado, las mujeres son mayoritariamente las principales cuidadoras, como se evidencia en la tabla 5 (25). 
TABLA 5

Porcentaje de cuidadores remunerados y no remunerados en salud por sexo. Total país, 2013

\begin{tabular}{|c|c|c|c|c|c|c|}
\hline & \multicolumn{2}{|c|}{ Varones } & \multicolumn{2}{c|}{ Mujeres } & \multicolumn{2}{c|}{ Total } \\
\cline { 2 - 7 } & Cantidad & $\%$ & Cantidad & $\%$ & Cantidad & $\%$ \\
\hline $\begin{array}{c}\text { Cuidadores } \\
\begin{array}{c}\text { Personales a } \\
\text { domicilio (5322) } \\
\text { remunerados }\end{array}\end{array}$ & 501 & 4.1 & 11794 & 95.9 & 12295 & 100 \\
\hline $\begin{array}{c}\text { Cuidadores no } \\
\text { remunerados en salud }\end{array}$ & 32258 & 25.6 & 93789 & 74.4 & 126047 & 100 \\
\hline
\end{tabular}

Fuente: reelaboración de [25].

Estos datos son consistentes con la evidencia con la que se cuenta a partir de los estudios de cuidados: las mujeres son las que se encargan mayoritariamente de su realización.

En cuanto a la estructura de edades de las personas cuidadoras en salud a cambio de una remuneración, vemos que se concentran en el tramo entre los 46 y los 65 años (51\%), lo que coincide con el perfil de quienes realizan trabajados no remunerados de cuidados en salud, ya que el $44.5 \%$ se ubica en el mismo tramo.

En este sentido, llama la atención el predominio de las personas de edades medias y más dentro de los cuidadores en salud. Sean remunerados o no remunerados, la mayoría (61.7\% y $63.9 \%$ respectivamente) son personas mayores de 45 años (tabla 6). 
TABLA 6

Cuidadores/as no remunerados y remunerados en salud por edad y sexo

\begin{tabular}{|c|c|c|c|c|c|c|}
\hline & \multicolumn{3}{|c|}{$\begin{array}{c}\text { Cuidadores/as no } \\
\text { remunerados en salud }\end{array}$} & \multicolumn{2}{c|}{$\begin{array}{c}\text { Cuidadores personales } \\
\text { en salud a domicilio } \\
\text { (5322) }\end{array}$} \\
\cline { 2 - 7 } & Varones & Mujeres & Total & Varones & Mujeres & Total \\
\hline $\begin{array}{c}\text { Entre 18 y 29 } \\
\text { años }\end{array}$ & 12.4 & 13.2 & 13 & 25.3 & 9 & 9.6 \\
\hline $\begin{array}{c}\text { Entre 30 y 45 } \\
\text { años }\end{array}$ & 43.3 & 36.4 & 38.2 & 8.8 & 26.6 & 25.8 \\
\hline $\begin{array}{c}\text { Entre 46 y 65 } \\
\text { Mayores de 66 }\end{array}$ & 13.5 & 8.7 & 9.9 & 15.2 & 12.8 & 12.9 \\
\hline Total & 100 & 100 & 100 & 100 & 100 & 100 \\
\hline
\end{tabular}

Fuente: reelaboración de [25].

En general, se encuentra que son las mujeres quienes realizan mayoritariamente tareas de cuidado no remunerado y remunerado en salud de edades avanzadas. En consistencia con los estudios de uso del tiempo, que miden el tiempo dedicado al trabajo no remunerado de cuidados, se presenta una fuerte feminización de estos, y como se ha planteado en la literatura, la realización de tareas de cuidado no remunerado presenta costos que impactan en la autonomía de las personas cuidadoras. Principalmente, las actividades de cuidado parecen generar procesos de exclusión de la participación o de participación diferencial entre varones y mujeres en el mercado laboral. Cuando se trata del cuidado dirigido a niños/as pequeños/as, se detecta que las mujeres cuidadoras tienen grandes conflictos para acceder y permanecer en el mercado laboral frente a sus pares varones [51]. Recurren entonces a diversas estrategias como reducir sus horas de trabajo en el mercado laboral, cambiar de empleos con horarios rígidos a empleos más flexibles, o bien excluirse completamente del mercado laboral. Respecto al cuidado por enfermedad, nótese en la tabla 7 que la mayoría de los cuidadores no remunerados en salud participa del mercado laboral (65.4\%), pero el porcentaje de mujeres que no lo hace es $14 \%$ más que los varones en esta situación. 
TABLA 7

Porcentaje de personas que cuidan por condición de actividad. Total país, 2013

\begin{tabular}{|c|c|c|c|}
\hline & Varones & Mujeres & Total \\
\hline $\begin{array}{c}\text { Desempeña una } \\
\text { actividad laboral }\end{array}$ & 75.8 & 61.8 & 65.4 \\
\hline $\begin{array}{c}\text { No desempeña una } \\
\text { actividad laboral }\end{array}$ & 24.2 & 38.2 & 34.6 \\
\hline Total & 100 & 100 & 100 \\
\hline
\end{tabular}

Fuente: reelaboración de [25].

Como se ha dicho, el cuidado de personas dependientes por motivos de enfermedad implica costos en la vida de los cuidadores. Cuando se observa la percepción sobre la limitación o modificación de las actividades diarias debido al cuidado no remunerado en salud por sexo del cuidador, son las cuidadoras mujeres las que declaran en menor medida que el cuidado no afecta en sus vidas (34.9\%), mientras que para el $42.2 \%$ de los varones el cuidado no remunerado en salud brindado a otras personas no afecta para nada sus actividades ni las limita (tabla 8). 
TABLA 8

Percepción sobre la limitación o modificación de actividades diarias debido al cuidado no remunerado en salud por sexo del cuidador/a

\begin{tabular}{|l|c|c|c|c|}
\hline & Varones & Mujeres & Total & $\begin{array}{c}\text { Diferencias } \\
\mathbf{M} / \mathbf{V}\end{array}$ \\
\hline Mucho & 28.3 & 31 & 30.3 & -2.7 \\
\hline Poco & 31.2 & 35 & 34 & -3.8 \\
\hline Nada & 40.5 & 34 & 35.7 & 6.5 \\
\hline Total & 100 & 100 & 100 & \\
\hline
\end{tabular}

Fuente: reelaboración de [25].

Entre aquellos cuidadores que declaran tener mucho o poco impacto en sus vidas cotidianas por la realización de cuidados no remunerados en salud, los principales recaen sobre el estudio, el trabajo remunerado o los ingresos, seguido de las redes y la recreación. En el primer ítem se expresan actividades que son fundamentales para la autonomía y que están en perjuicio tanto de varones como de mujeres ( 53.7 y 43.4\%, respectivamente). Es probable que los varones sientan este tipo de impacto más que las mujeres, debido a que estas suelen estar en contacto con la esfera doméstica, mientras que los varones pueden sentir un distanciamiento mayor de la esfera pública (del trabajo y el estudio) que las mujeres por motivos de cuidados en salud (tabla 9). 
TABLA 9

Sexo de los/as cuidadores/as según tipo de impacto. Total país, 2013

\begin{tabular}{|c|c|c|c|}
\hline & Varones & Mujeres & Total \\
\hline $\begin{array}{c}\text { Estudio/trabajo } \\
\text { remunerado/ingresos }\end{array}$ & 53.7 & 43.4 & 45.8 \\
\hline Redes y recreación & 19 & 22.6 & 21.8 \\
\hline Salud personal & 13.2 & 16.7 & 15.9 \\
\hline Convivencia & 7.4 & 9 & 8.6 \\
\hline Ninguno & 6.6 & 7 & 6.9 \\
\hline No sabe & 0 & 1.2 & 0.9 \\
\hline Total respuestas & 100 & 100 & 100 \\
\hline
\end{tabular}

Fuente: reelaboración de [25].

Los datos ponen de manifiesto cómo en el marco de los hogares son las mujeres las que específicamente se dedican a cuidar de manera no remunerada en salud y cómo también son ellas las que tienen una percepción de impactos negativos sobre su vida mayor que la de los varones.

Sumado a esto, los hogares (particularmente las mujeres dentro de estos) no solo brindan cuidados a sus integrantes, sino que muchas veces brindan cuidados a personas que no pertenecen al hogar (tabla 10). 
TABLA 10

Tipo de vínculo entre beneficiario/a y cuidador/a, por sexo del cuidador/a. Total país, 2013

\begin{tabular}{cccc}
\hline & Varones & Mujeres & Total \\
\hline $\begin{array}{c}\text { Relación de parentesco, con } \\
\text { integrante del hogar }\end{array}$ & 63 & 55.7 & 57.6 \\
\hline $\begin{array}{c}\text { Relación de parentesco, } \\
\text { fuera del hogar }\end{array}$ & 21.7 & 39 & 34.6 \\
\hline $\begin{array}{c}\text { No parientes fuera del } \\
\text { hogar }\end{array}$ & 9 & 4.5 & 5.6 \\
\hline $\begin{array}{c}\text { No parientes que integran } \\
\text { el hogar }\end{array}$ & 6.3 & 0.8 & 2.2 \\
\hline Total & 100 & 100 & 100 \\
\hline
\end{tabular}

Fuente: reelaboración de [25].

En relación con el porcentaje de hogares que realizan cuidados no remunerados en salud, estos representan un número menor al de hogares que realizan cuidados en general. Como muestran los datos de la Encuesta de Uso del Tiempo 2013 [52], el 38.1\% de los hogares de Uruguay realiza tareas no remuneradas de cuidado (a niños, personas mayores o discapacitados en situación de dependencia), mientras que los datos de la Encuesta Nacional de Cuidados No Remunerados en Salud muestran que es el 17.5 \% de los hogares en el país el que se dedica a tareas de cuidado no remunerado en salud. Esta baja en el porcentaje es producto de la especificidad que tienen los cuidados no remunerados en salud: para estar ante la presencia de un hogar cuidador debe haber alguien con alguna enfermedad (crónica o aguda), lo que establece el requerimiento de que haya una situación de dependencia en el hogar (enfermedad), con menor frecuencia que otras y, según el tipo, más versátil también (tabla 11). 
TABLA 11

Porcentaje de hogares cuidadores no remunerados y cuidadores no remunerados en salud según Encuesta de Uso del Tiempo (EUT) y ENCNRS 2013

\begin{tabular}{|c|c|c|c|c|c|}
\hline \multicolumn{3}{|c|}{ EUT 2013} & \multicolumn{3}{|c|}{ ENCNRS 2013} \\
\hline \multirow{3}{*}{$\begin{array}{c}\text { Cuida sin } \\
\text { remuneración a } \\
\text { cambio }\end{array}$} & $\mathrm{Si}$ & 38.1 & \multirow{3}{*}{$\begin{array}{l}\text { Cuida sin } \\
\text { remuneración a } \\
\text { cambio en salud }\end{array}$} & Si & 17.5 \\
\hline & No & 61.9 & & No & 82.5 \\
\hline & Total & 100 & & Total & 100 \\
\hline
\end{tabular}

Fuente: elaboración propia con base en los microdatos de la Encuesta de Uso del Tiempo.

Por el mismo motivo, se observa que la carga de tiempo dedicado al cuidado no remunerado en salud es mayor al tiempo promedio general dedicado a los cuidados (tabla 11). Este aspecto tiene que ver con la intensidad del cuidado en salud en el marco de los hogares, ya que ante una situación de enfermedad de un integrante del hogar aumenta considerablemente la demanda de disposición y tiempo de los/as cuidadores/ as (tabla 12).

TABLA 12

Promedio semanal de tiempo de cuidados en EUT y ENCNRS 2013

\begin{tabular}{|c|c|c|}
\hline & Varones & Mujeres \\
\hline ENCNRS & 46 & 57 \\
\hline $\begin{array}{c}\text { EUT (total de los } \\
\text { cuidados) }\end{array}$ & 35 & 24 \\
\hline
\end{tabular}

Fuente: elaboración propia con base en los datos de la ENCNRS y EUT, 2013 (INE).

Por último, sumado a que las mujeres son las mayores proveedoras de cuidados no remunerados en salud, ellas son las que tienen una mayor tasa de participación en los hogares donde se prestan cuidados no remunerados en salud (55\%) en comparación con los varones (22\%).

Entre las mujeres, dentro de los componentes del cuidado no remunerado en salud, la tasa de participación más alta se encuentra en los cuidados específicos en salud, caracterizados por ser rutinarios y requerir producción. Por su parte, la mayor tasa de participación de los varones es en las tareas de acompañamiento, en las que se puede contar con mayor flexibilidad y suelen ser tareas de 'apoyo' y no de producción directa.

En este sentido, es posible hipotetizar sobre la presencia de una diferenciación del cuidado no remunerado en salud cuantitativa entre varones y mujeres, pero también cualitativa, que también se evidencia en otros tipos de cuidados no remunerados (tabla 13). 
TABLA 13

Tasa de participación y tiempo promedio dedicado a los cuidados no remunerados en salud y cada uno de sus componentes por las personas cuidadoras. Total país, 2013

\begin{tabular}{|c|c|c|c|c|c|c|}
\hline & \multicolumn{2}{|c|}{ Mujeres } & \multicolumn{2}{c|}{ Varones } & \multicolumn{2}{c|}{ Diferencias MV } \\
\cline { 2 - 8 } & $\begin{array}{c}\text { Tasa de } \\
\text { participación }\end{array}$ & $\begin{array}{c}\text { Horas } \\
\text { semanales } \\
\text { promedio }\end{array}$ & $\begin{array}{c}\text { Tasa de } \\
\text { participación }\end{array}$ & $\begin{array}{c}\text { Horas } \\
\text { semanales } \\
\text { promedio }\end{array}$ & $\begin{array}{c}\text { Tasa de } \\
\text { participación }\end{array}$ & $\begin{array}{c}\text { Horas } \\
\text { semanales } \\
\text { promedio }\end{array}$ \\
\hline $\begin{array}{c}\text { Cuidados específicos en } \\
\text { salud }\end{array}$ & 47 & 22 & 16 & 21 & 32 & 2 \\
\hline $\begin{array}{c}\text { Cuidados de apoyo } \\
\text { Acompañamiento }\end{array}$ & 40 & 22 & 15 & 18 & 25 & 3 \\
\hline $\begin{array}{c}\text { Compras para la } \\
\text { enfermedad y gestiones con } \\
\text { el sistema de salud }\end{array}$ & 28 & 4 & 12 & 6 & 17 & -1 \\
\hline $\begin{array}{c}\text { Cuidados no remunerados } \\
\text { en salud }\end{array}$ & 55 & 57 & 22 & 46 & 33 & 12 \\
\hline
\end{tabular}

Fuente: elaboración propia con base en los datos de la ENCNRS, 2013.

Finalmente, dentro de las tareas a las que se dedican más horas en promedio, tanto para varones como para mujeres se presentan las tareas de acompañamiento, seguidas por los cuidados específicos y de apoyo. En las tareas de acompañamiento es en las que se encuentra la mayor brecha de tiempo dedicada entre mujeres y varones, seguidas por los cuidados de apoyo. En el total de los cuidados no remunerados en salud se observa una brecha de doce horas semanales entre varones y mujeres.

\section{Reflexiones finales}

El aporte de las ciencias sociales a la reformulación de los conceptos de trabajo, producción y en la valorización y medición de estas actividades permite visibilizar desigualdades de género imperantes, que, desde una fragmentada división sexual del trabajo en el marco de los hogares, pasando por una clara feminización de los trabajos de cuidado remunerados, cuestiona concepciones tradicionales y sus derivaciones metodológicas por contar con un doble sesgo: androcéntrico y mercantil.

Los cuidados no remunerados en salud son un aporte fundamental a la salud de la población, y son específicamente un aporte de los hogares a la economía, en la medida que producen bienes y servicios altamente significativos para el bienestar social.

Las mujeres son quienes trabajan más horas ( 57 vs. 46 ) y de mayor manera (representan el $74.4 \%$ de las personas cuidadoras, con una tasa de participación del 55\% en el marco de los hogares). Se ha mostrado que esto tiene costos negativos asociados para su autonomía y ejercicio de la ciudadanía social: disponen de poco tiempo, trabajan menos que las personas que no cuidan, perciben efectos negativos en su salud, en su posibilidad de socializar con amigos o redes vecinales, en sus posibilidades de estudiar y trabajar y también en la disponibilidad de tiempo personal.

Sabemos, por ejemplo, que las tareas de cuidado que apartan a las mujeres del mercado laboral, las apartan también de los derechos sociales que están canalizados a través de la participación en dicho mercado 
(cuando es formal). También, que esto tiene consecuencias presentes en su autonomía económica actual (disponibilidad de recursos) y futura (las pensiones y jubilaciones serán menores durante la vejez en función de la participación laboral).

Las restricciones en la disponibilidad de tiempo por dedicarlo a las tareas de cuidado han de ser tenidas en cuenta, sobre todo cuando se piensa en la organización social de los cuidados. Es sin duda imperante avanzar hacia un modelo, en este caso, de cuidados en salud que no descanse sobre el trabajo gratuito de las mujeres en el marco de los hogares.

Por otra parte, el trabajo remunerado de cuidados en salud está igualmente feminizado, con lo cual podemos establecer que la mercantilización de los cuidados en salud reproduce la división sexual del trabajo que establece que son las mujeres las que se han de encargar de los cuidados.

Se visualiza la fortaleza del sistema de género imperante que se articula virtuosamente con el capitalismo y no innova en los roles, fomentando una mayor participación masculina, ya sea en los cuidados remunerados, o bien en los no remunerados.

Otro de los datos relevantes tiene que ver con la división sexual del trabajo dentro de los cuidados no remunerados en salud. Dentro de estos existe una brecha de dedicación de tiempo semanal entre mujeres y varones de doce horas y hemos mostrado que ellas participan más. Pero, sumando a esto, observamos una distinción cualitativa entre los tipos de trabajo en los que participan más las mujeres y los varones. Ellas participan en tareas rutinarias y obligatorias como son los cuidados específicos en salud (hacer tratamientos - por ejemplo, curaciones o inyecciones-, controlar manifestaciones de la enfermedad — control de la temperatura-, administrar medicinas). Mientras tanto, los varones participan de forma mayoritaria en tareas más laxas y no "obligatorias" como son las tareas de acompañamiento (entretenimiento, acompañamiento a consultas médicas, traslados entre otras). Esta división cuantitativa y cualitativa en que las mujeres participan más, dedican más tiempo y se dedican a las tareas vinculadas a la producción directa de los servicios o los bienes, rutinarias y "obligatorias" para que el cuidado del otro cumpla su función también es frecuente en los cuidados infantiles, a adultos mayores y discapacitados. La asociación cultural de las mujeres como las indicadas naturalmente para realizar este tipo de trabajo, probablemente tenga que ver con su permanencia en estos roles de cuidadoras y en las tareas que más tiempo y orden (como restricciones) les demandan.

En cuanto a la valorización del trabajo de cuidados no remunerados en salud, se muestra que estos representan un $1.8 \%$ del PIB nacional para 2013 y un 32.2\% sobre el agregado del sector salud. Esto implica que, en caso de no existir el trabajo gratuito de los hogares, y particularmente de las mujeres en este marco, el estado o el mercado deberían producir un tercio más del total de bienes y servicios que aportan actualmente a la economía.

Una vez más, se evidencia la esencialidad del rol de los hogares y del trabajo femenino no remunerado en la provisión de bienes y servicios para la sociedad. La centralidad de su aporte y los potenciales costos del cuidado en la autonomía de las mujeres ponen sobre la mesa la pregunta acerca de la organización social del cuidado en Uruguay, mientras que recuerdan que tanto el reconocimiento como la redistribución del trabajo no remunerado sigue siendo imperantes.

Todos estos elementos son insumos para poder pensar políticas públicas que busquen el logro de un equilibrio mayor entre los actores que pueden aportar al bienestar social y a la equidad de género. El desafío llega en un momento crucial, en la medida en que desde el Estado uruguayo se puede pensar en la articulación del floreciente Sistema Nacional de Cuidados con el Sistema Nacional Integrado de Salud.

\section{Agradecimientos}

El presente proyecto es parte de una investigación que fue financiada entre 2012 y 2014 por la Comisión Sectorial de Investigación Científica de la Universidad de la República Oriental del Uruguay y contó con apoyos económicos de la División de Asuntos de Género de la Comisión Económica para América Latina 
y el Caribe (Cepal), en el marco del proyecto Mejorando la cuantificación del trabajo no remunerado de las mujeres para las politicas de erradicación de la pobreza.

\section{Referencias}

1. Tronto J, Fisher B. Toward a feminist theory of caring. En Abel EK, Nelson MK. Circles of care: work and identity in women's lives. Albany, Nueva York: State University of New York Press; 1990. p. 36-54.

2. Aguirre R. Uso del tiempo y desigualdades de género en el trabajo no remunerado En Aguirre R, editora. Las bases invisibles del bienestar social. El trabajo no remunerado en Uruguay. Montevideo: Doble Clic Editoras; 2009.

3. Hochschild A. The managed heart. California: University of California Press; 1983.

4. Esping-Andersen G. Fundamentos sociales de las economías postindustriales. Barcelona: Ariel Sociología; 2000.

5. Carrasco C. El trabajo de las mujeres: producción y reproducción. Apuntes para su reconceptualización. Cuadernos de Economía. 1992; (20):95-109.

6. Bock G, Duden B. Trabajo por amor, amor como trabajo. Desarrollo. Revista de la Sociedad Internacional para el Desarrollo. 1985; (2):4-14.

7. Durán MA. De puertas adentro. Madrid: Ministerio de Cultura, Instituto de la Mujer; 1988.

8. Aguirre R. Las bases invisibles del bienestar social. El trabajo no remunerado en Uruguay. Montevideo: Doble Clic Editoras; 2009.

9. Gómez Luna ME. Cuenta satélite de los hogares: valoración del trabajo doméstico no pagado. El caso de México. Versión preliminar del trabajo presentado en el Taller internacional Cuentas Nacionales de Salud y género. Santiago de Chile: OPS-OMS-Fonasa; 2001.

10. Adelantado J, et al. Las relaciones entre estructura y políticas sociales: una propuesta teórica. Revista Mexicana de Sociología. 1998; (3):123-56.

11. Daly M, Lewis J. El concepto de social care y el análisis de los estados de bienestar contemporáneos. En Carrasco C. Borderías C, Torns T, editores. El trabajo de cuidados: Historia, teorías y política. Madrid: Los Libros de la Catarata; 2011.p. 225-52.

12. Aguirre R. Género, ciudadanía social y trabajo. Montevideo: Doble Clic Editoras; 2003.

13. Martínez J, Voorend K. Desigualdades de género en los regímenes de bienestar latinoamericanos: mercado, política social y organización familiar de los cuidados En Pautassi L, Ziebecchi C, editores. Las fronteras del cuidado. Agenda, derechos e infraestructura. Buenos Aires: Editorial Biblos; 2010.

14. Aguirre R. Uso del tiempo y desigualdades de género en el trabajo no remunerado En Aguirre R, editora. Las bases invisibles del bienestar social. El trabajo no remunerado en Uruguay. Montevideo: Doble Clic Editoras; 2009.

15. Batthyány K, Genta N, Perrotta V. Trabajo no remunerado y cuidados. Primeros resultados de la Encuesta de Uso del Tiempo 2013. En Boado M, coordinador. El Uruguay desde la sociología 13. Montevideo: DS-FCS-Udelar; 2015.

16. Aguirre R. Personas ocupadas en el sector cuidados. Montevideo: Serie Sistema Nacional de Cuidados; 2013.

17. Anderson J. Sistemas de género y procesos de cambio. En Batthyány K, coordinadora. Género y desarrollo. Una propuesta de formación. Montevideo: Doble Clic Editoras; 2006.

18. Arriagada I. Familias latinoamericanas: convergencias y divergencias de modelos y políticas. Revista de la Cepal. 1998; (65):85-102.

19. Beck U, Beck-Gernsheim E. Amor a distancia. Nuevas formas de vida en la era global. Buenos Aires: Paidós Contextos; 2012.

20. Batthyány K, Genta N, Perrotta V. La población uruguaya y el cuidado: análisis de representaciones sociales y propuestas para un sistema de cuidados en Uruguay. Montevideo: Ministerio de Desarrollo Social; 2013.

21. Montaño S. El cuidado en acción. En Monaño S, Calderón C, editores. El cuidado en acción: entre el derecho y el trabajo. Santiago de Chile: Cepal-Unifem, Cuadernos de la Cepal N.o 94; 2010. 
22. Himmelweit $S$. El descubrimiento del trabajo no remunerado: consecuencias sociales de la expansión del término trabajo. En Carrasco C, Borderías C, Torns T, editoras. El trabajo de cuidados. Historia, teoría y políticas. Madrid: Editorial Catarata; 2011.

23. Carrasco C. El trabajo de las mujeres: producción y reproducción. Apuntes para su reconceptualización. Cuadernos de Economía. 1992; (20):95-109.

24. Legarreta M. El tiempo donado en el ámbito doméstico. Reflexiones para el análisis del trabajo doméstico y los cuidados En Arango Gaviria LG, Molinier P, editoras. El trabajo y la ética del cuidado. Medellín: La Carreta Editores; 2011.

25. Batthyány K, Genta N, Perrotta V. Trabajo no remunerado y cuidados. Primeros resultados de la Encuesta de Uso del Tiempo 2013. En Boado M, coordinadores. El Uruguay desde la sociología 13. Montevideo: DS-FCS-Udelar; 2015.

26. Rogero García J. Los tiempos del cuidado. El impacto de la dependencia de los mayores en la vida cotidiana de sus cuidadores. Madrid: Publicaciones Imserso; 2010.

27. García-Calvente MM, Mateo-Rodríguez I, Eguiguren A. El sistema informal de cuidados en clave de desigualdad. Gaceta Sanitaria. 2004; (18):132-9.

28. Salleras Sanmartí L. La salud y sus determinantes. En Salleras Sanmartí L. Educación sanitaria. Madrid: Ediciones Díaz de Santos; 1985.

29. Contrandiopoulos A. Elementos para una topografía del concepto de salud. Ruptures. Revista Interdisciplinaria de la Salud. 2006; 11(1):86-99.

30. Salleras Sanmartí L. La salud y sus determinantes. En Salleras Sanmartí L. Educación sanitaria. Madrid: Ediciones Díaz de Santos; 1985.

31. Rogero García J. Los tiempos del cuidado. El impacto de la dependencia de los mayores en la vida cotidiana de sus cuidadores. Madrid: Publicaciones Imserso; 2010.

32. Batthyány K, Genta N, Perrotta V. El aporte de las familias y las mujeres al cuidado no remunerado de la salud en Uruguay. Santiago de Chile: Cepal; 2015.

33. Gómez Luna ME. Cuenta satélite de los hogares: valoración del trabajo doméstico no pagado. El caso de México. Versión preliminar del trabajo presentado en el Taller internacional Cuentas Nacionales de Salud y género. Santiago de Chile: OPS-OMS-Fonasa; 2001.

34. García-Calvente MM, Mateo I, Gutiérrez P. Cuidados y cuidadores en el sistema informal de salud. Granada: Escuela Andaluza de Salud Pública e Instituto Andaluz de la Mujer; 1999.

35. Durán MA. Los costos invisibles de la enfermedad. Madrid: Fundación BBV; 2003.

36. Durán MA. Diez buenas razones para medir el trabajo no remunerado en el cuidado de la salud. En La economía invisible y las desigualdades de género. La importancia de medir y valorar el trabajo no remunerado. Washington: OPS; 2008.

37. García-Calvente MM, Mateo I, Gutiérrez P. Cuidados y cuidadores en el sistema informal de salud. Granada: Escuela Andaluza de Salud Pública e Instituto Andaluz de la Mujer; 1999.

38. García-Calvente MM, Mateo-Rodríguez I, Eguiguren A. El sistema informal de cuidados en clave de desigualdad. Gaceta Sanitaria. 2004; (18):132-9.

39. Cuidados en la vejez. El apoyo informal. Madrid: Inserso; 1995.

40. La Parra D. Contribución de las mujeres y los hogares más pobres a la producción de cuidados de salud informales Gaceta Sanitaria. 2001; 15(6):498-05.

41. Hernández Bello A. El trabajo no remunerado de cuidado de la salud: naturalización e inequidad. Revista Gerencia y Políticas de Salud. 2009; 8(17):173-85.

42. Ferrán L. Marco conceptual y lineamientos metodológicos de la cuenta satélite de los hogares para medir el trabajo no remunerado en salud. En OPS, coordinador. La economía invisible y las desigualdades de género. La importancia de medir y valorar el trabajo no remunerado. Washington: OPS; 2008. 
43. Batthyány K, Genta N, Perrotta V. El aporte de las familias y las mujeres al cuidado no remunerado de la salud en Uruguay. Santiago de Chile: Cepal; 2015.

44. Salvador S. La valoración económica del trabajo no remunerado. En Aguirre R, editor. Las bases invisibles del bienestar social. El trabajo no remunerado en Uruguay. Montevideo: Doble Clic Editoras; 2009.

45. Anderson J. Sistemas de género y procesos de cambio. En Batthyány K, coordinadora. Género y desarrollo. Una propuesta de formación. Montevideo: Doble Clic Editoras; 2006.

46. Salvador S. La valoración económica del trabajo no remunerado. En Aguirre R, editor. Las bases invisibles del bienestar social. El trabajo no remunerado en Uruguay. Montevideo: Doble Clic Editoras; 2009.

47. Salvador S. La valoración económica del trabajo no remuenrado. En Batthyány K, editor. Los tiempos del bienestar social. Género, trabajo no remunerado y cuidados en Uruguay. Montevideo: Doble Clic Editoras; 2015.

48. Batthyány K, Genta N, Perrotta V. El aporte de las familias y las mujeres al cuidado no remunerado de la salud en Uruguay. Santiago de Chile: Cepal; 2015.

49. Salvador S. La valoración económica del trabajo no remunerado. En Aguirre R, editor. Las bases invisibles del bienestar social. El trabajo no remunerado en Uruguay. Montevideo: Doble Clic Editoras; 2009.

50. Espino A, et al. La economía de los hogares: más allá de los ingresos monetarios. Montevideo: Ciedur, ONUMujeres; 2010.

51. Diagnóstico de las problemáticas de las madres con hijos pequeños para acceder o permanecer en el mercado laboral. México: Sedesol; 2010.

52. Encuesta de Uso del Tiempo y del Trabajo No Remunerado (EUT). Instituto Nacional de Estadística. Montevideo: INE- MIDES-INMUJERES-FCS; 2013.

\section{Notas}

* Artículo de investigación.

\section{Licencia Creative Commons CC BY 4.0}

Como citar este artículo: Batthyány Dighiero K, Scavino Solari S. Valorización económica de los cuidados no remunerados en salud: un aporte al reconocimiento del trabajo invisible de los hogares y las mujeres. Rev Gerenc Polít Salud. 2017; 17(34): 1-23. https://doi.org/10.11144/Javeriana.rgps17-34.vecr 